\title{
Note from Editor-in-Chief
}

\section{Dolf Weijers}

Published online: 3 May 2013

(C) Springer-Verlag Berlin Heidelberg 2013

Plants devote much of their energy to generate reproductive structures and to form them in the right place and at the most appropriate time. While reproduction in plants culminates in the formation of an embryo and seed, many critical steps precede this. In order to arrive at a complete understanding of the genetic or environmental control of plant reproduction, we should therefore consider not only the "supreme moment" of fertilization, but also the steps leading up to this event, as well as the processes that turn the product of fertilization into a healthy, mature seed.

Plant Reproduction strives to be a prime outlet for important new insights in the processes underlying successful plant reproduction in the broadest sense. With the change of name comes a change of scope. While the former journal title suggested a focus only on those events related to the sexual process, we now explicitly welcome contributions to the understanding of plant reproduction in general. An excellent example is the paper by Ogawa et al. (2013) in this issue, in which the genetic basis for different components of asexual (apomictic) reproduction in Hieracium is illuminated. Given the fast-paced developments in the field of flowering and flower development, we also hope to receive more high-quality manuscripts in this area, which we aim to include in the journal's wider scope.

Another important change in the policy of the journal is that there will be an emphasis on mechanistic rather than entirely descriptive studies. Obviously, there is still much to learn from observing normal reproductive processes, as exemplified by the papers by Lawit et al. (2013) and by Leshem et al. (2013) in this issue. Both studies use

D. Weijers $(\square)$

Laboratory of Biochemistry, Wageningen University,

Dreijenlaan 3, 6703 HA Wageningen, The Netherlands

e-mail: dolf.weijers@wur.nl molecular markers to reveal previously unidentified aspects of fertilization (Leshem et al. 2013) or to visualize at high resolution and molecular scale the events associated with cell fate specification in the female gametophyte (Lawit et al. 2013). These two studies make important new contributions, despite being largely descriptive. Importantly, as the model species Arabidopsis is used, the findings in these two papers contribute to a large pre-existing body of data. When using non-model organisms, Plant Reproduction will give priority to those manuscripts that describe an advance that is likely to be of more general interest. In addition to the information gained from observation and description, important insights are gained from perturbation. Therefore, studies that provide information on underlying mechanisms based on, for example, genetic perturbation are also strongly encouraged.

The field of plant reproduction research has made amazing strides over the last few decades and is firmly grounded in strong scientific foundations. The field deserves a dedicated journal that publishes important advances. Plant Reproduction will strive to be this journal, and I look forward to publishing exciting science.

\section{References}

Lawit SJ, Chamberlin MA, Agee A, Caswell ES (2013) Transgenic manipulation of plant embryo sacs tracked through cell-type-specific fluorescent markers: cell labeling, cell ablation, and adventitious embryos. Plant Reprod. doi:10.1007/s00497-013-0215-x

Leshem Y, Johnson C, Sundaresan V (2013) Pollen tube entry into the synergid cell of Arabidopsis is observed at a site distinct from the filiform apparatus. Plant Reprod. doi:10.1007/s00497-013-0211-1

Ogawa D, Johnson SD, Henderson ST (2013) Genetic separation of autonomous endosperm formation (AutE) from the two other components of apomixis in Hieracium. Plant Reprod. doi: 10.1007/s00497-013-0214-y 